\title{
L'aferesi terapeutica oggi
}

\section{Gaspare Elios Russo', Silvia Lai ${ }^{2}$, Massimo Testorio ${ }^{1}$, Anna Rita D’Angelo ${ }^{1}$, Andrea Martinez, Alessandra Nunzi ${ }^{1}$, Virgilio DeBono ${ }^{1}$, Dmytro Grynyshyn ${ }^{1}$, Tania Gnerre Musto ${ }^{1}$}

\author{
${ }^{1}$ Dipartimento di Scienze ginecologico-ostetriche e urologiche, Sapienza, Università di Roma, Roma \\ ${ }^{2}$ Dipartimento di Medicina Clinica, Sapienza, Università di Roma, Roma
}

THERAPEUTIC PLASMAPHERESIS TODAY

Abstract. In 1914, Abel, along with Rowentree and Turner, introduced the term "plasmapheresis" whose literal meaning is "subtraction". The first "therapeutic plasmapheresis" was performed in 1952 in a patient with multiple myeloma; in 1963, patients with paraproteinemia were treated with plasmapheresis to reduce blood hyperviscosity, as described by Salomon and Fahey. During the course of time, more specific and selective techniques were gradually introduced, allowing the use of the treatment for new clinical indications (plasma exchange, CRYO apheresis, leukapheresis, thrombocytopheresis, lymphocyte apheresis, LDL apheresis). The current indications for plasmapheresis have been defined, and periodically re-established, by two scientific American associations, the American Association of Blood Banks (AABB) and the American Society of apheresis (ASFA), based on the available evidence of efficacy of this treatment in specific diseases. In 1993, a study group for therapeutic apheresis was created within the Italian Society of Nephrology, and was also aimed at developing new guidelines. The aim of therapeutic apheresis is to remove pathogenic substances. The use of techniques for selective removal allows a lesser elimination of non-pathological components, reducing the risk of infections, bleeding, and allergic reactions. However, although the therapeutic success is related to the amount of the pathological substances removed, it is likely that plasma also acts by modulating the patients' immune system. In fact, normal apheretic procedures would act directly on the immune system's components and mediators. Therapeutic apheresis is indicated during the course of immunological disorders including dermatological, hematological, oncological, dysmetabolic, neurological, and renal diseases, and is also used in an emergency setting as a technique for the detoxification of both endogenous and exogenous substances to avoid irreversible organ damage. Therapeutic plasmapheresis has undergone significant changes due to the development of the equipment and to the new indications. In fact, the technological innovation has introduced new methods that allow plasmapheresis to be more tolerable and less invasive. Hemofenix uses filtration through a membrane with an innovative system of nanofiltration, the ROSA filter. Hemofenix allows to perform the treatment with a single, small needle and with a greatly reduced extracorporeal volume of about $70 \mathrm{~mL}$, thus reducing the risks for the patients, even in children. Additional advantages are represented by the low priming and short duration of the treatment; furthermore, the exchange of low volumes during the treatment allows not using plasma as replacement fluid, thus reducing the risk of infections and allergic reactions related to plasma exchange. Clinical trials should be performed to demonstrate the effectiveness of this method by comparing this therapy with the traditional apheretic one.

Key words: Therapeutic apheresis, Nanofiltration, Hemofenix, ROSA filter

Conflict of interest: None.

Financial support: None.

Accettato: 7 Marzo 2014

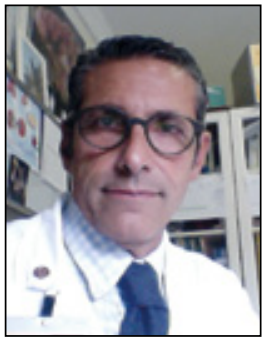

Gaspare Elios Russo
L'aferesi o aphàiresis, parola di origine greca il cui significato letterale è "sottrazione", è oggi considerata come una forma raffinata dell'antico salasso. "Come da un pozzo si estrae acqua torbida fino a che dalla sorgente sotterranea non venga acqua pura soltanto, cosi va cavato sangue corrotto fino a che non rimanga altro che quello puro" affermava Botallo già nel 1500 .

L'aferesi terapeutica trae le sue origini nel Medioevo, periodo in cui il salasso veniva impiegato in varie situazioni con l'intento di ottenere, attraverso tale terapia, la rimozione degli "umori cattivi", ed è stata ampiamente utiliz- zata fino al XIX secolo per trattare un'ampia varietà di condizioni patologiche (1). Nel 1914 Abel, insieme a Rowentree e a Turner, introdusse, per primo, il termine di "plasmaferesi" con la pubblicazione di Plasma removal with return of corpuscles (plasmaphaeresis). Abel può, così, essere considerato a tutti gli effetti il padre della Dialisi e dell'Aferesi. La prima "plasmaferesi terapeutica" risale al 1952 in un paziente affetto da mieloma multiplo, ma occorre aspettare fino al 1963 per avere le prime vere applicazioni cliniche in soggetti affetti da paraproteinemia per ridurre l'iperviscosità del sangue, a opera di Salomon e Fahey $(2,3)$. Intorno agli anni '60 si ha la comparsa delle prime attrezzature automatiche: i cosiddetti separatori cellulari, che permettevano lo scambio di elevate quantità di plasma in poche ore. Nel tempo sono stati progressivamente 


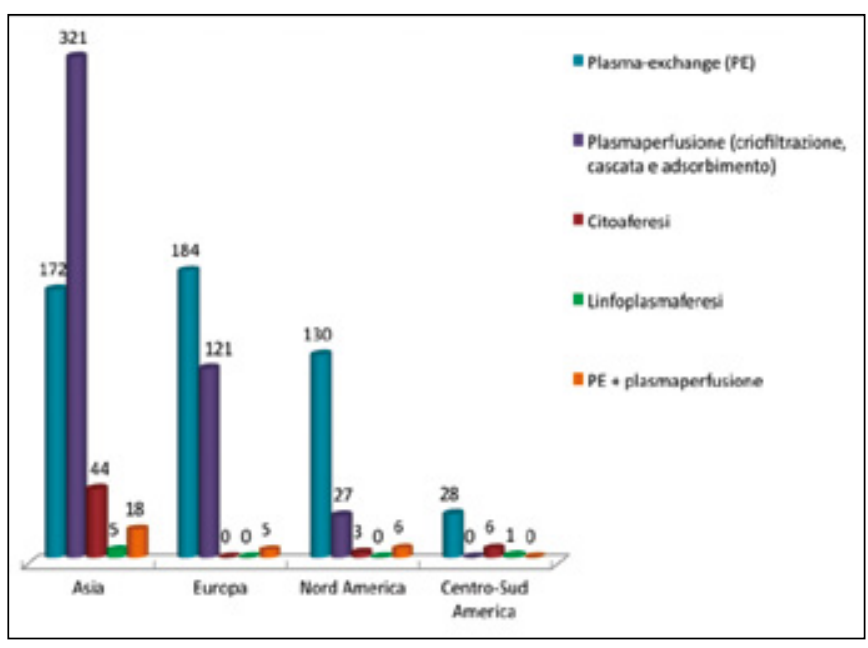

Fig. 1 - percentuale di pazienti trattati con diversi tipi di trattamenti aferetici divisi per aree geografiche.

introdotti termini sempre più specifici a seconda delle modalità e del campo di applicazione della metodica, dell'evoluzione dei materiali d'uso, della diversificazione delle tecniche e delle possibilità di impiego clinico (PLASMA-EXCHANGE, CRIOAFERESI, LEUCOAFERESI, TROMBOCITOAFERESI, LINFOCITOAFERESI, LDL-AFERESI) (3, 4). Nella Figura 1 viene mostrata la frequenza di impiego delle diverse tecniche aferetiche nei diversi continenti, aggiornata al 2010. Oggi, il termine "Emaferesi" ha sostituito i precedenti definendo la procedura con cui si vuole ottenere la riduzione o la rimozione di selettive linee cellulari (eritrociti, granulociti, linfociti, piastrine) o di plasma o di componenti plasmatici prodotti in eccesso o con caratteristiche patologiche o di sostanze tossiche comunque introdotte $(4,5)$.

\section{Indicazioni attuali}

Oggi la plasmaferesi rappresenta un presidio terapeutico assai complesso che richiede specifiche conoscenze e competenze con un'ampia varietà di campi di impiego (reumatologia, nefrologia, ematologia, ecc.). Pertanto, essendo diverse le discipline coinvolte, esse potrebbero cooperare dando il loro apporto alla stesura di un testo per l'appropriata gestione della metodica. In Italia, come nel resto dell'Europa, questa attività clinica è stata gestita in maniera poco attenta, tanto che, a oggi, non si riconoscono né degli specifici ambiti di impiego né $i$ settori di utenza. In assenza di una specifica o univoca indicazione terapeutica, si rende necessario l'apporto di competenze dei diversi specialisti che, attraverso l'utilizzo di registri nazionali e internazionali e la progettazione di trial, possano favorire la stesura di Linee Guida. Ancora oggi, le uniche Linee Guida per l'aferesi terapeutica sono quelle definite e periodicamente ristabilite da due associazioni scientifiche americane: l'American Association of Blood Banks (AABB) e l'American Society of Apheresis (ASFA), sulla base delle evidenze disponibili di efficacia del trattamento in specifiche malattie. Le Linee Guida ASFA sono state introdotte nel 1986, modificate in parte nel 1993 e poi aggiornate nel 1996, nel 2000 e nel 2013 (6-8). Nel 1993, si è costituito, in seno alla Società Italiana di Nefrologia, il gruppo di studio dell'aferesi terapeutica, che si prefiggeva il compito di censire tutte le strutture, in ambito nefrologico, che utilizzavano o intendevano utilizzare la plasmaferesi terapeutica e sviluppare Linee Guida di riferimento. Il fine ultimo della terapia aferetica sarebbe quello di poter rimuovere solo le sostanze patogene. L'uso di tecniche di rimozione selettiva si accompagna in realtà non tanto a una maggiore capacità di estrazione della sostanza, bensì a una minore rimozione di componenti non patologiche (8-10). La riduzione delle immunoglobuline non patogene, per esempio, potrebbe predisporre a infezioni, specialmente in soggetti a rischio come, per esempio, quelli sottoposti a terapia immunosoppressiva o con insufficienza renale. Tuttavia, sebbene il successo terapeutico sia in parte legato alla quantità della sostanza patologica rimossa, è altresì probabile che la plasmaferesi agisca anche modulando il sistema immunitario dei pazienti: le normali procedure aferetiche sembrerebbero, infatti, intervenire sui componenti e sui mediatori del sistema immune e sull'evoluzione stessa del processo immunologico che verrà, di conseguenza, modulato in modo comunque indipendente e talvolta inaspettato rispetto alle nostre intenzioni (11-13).

Le domande poste nel 1600 da Burton che, nell'Anatomia della Melanconia, affermava che "Nell'operare il salasso bisogna considerare tre cautele: a chi farlo, quanto e quando farlo", restano ancora oggi attuali. Si spera che lo sviluppo di specifiche Linee Guida possa portare risposta a una serie di queste domande, ovvero: le patologie che possono godere della terapia aferetica, il razionale di utilizzo della metodica, i marker sierici sensibili e specifici per la guida e il controllo della procedura, lo stadio clinico idoneo per iniziare il trattamento, la scelta della tecnica più idonea, il numero di sedute e l'intervallo di tempo tra le stesse, il numero di cicli e l'intervallo tra gli stessi, il volume plasmatico da scambiare o processare e la terapia farmacologica da associare alla plasmaferesi terapeutica con il relativo dosaggio.

L'aferesi terapeutica trova indicazione in patologie di tipo immunologico-dermatologico, ematologico, oncologico, dismetabolico, neurologico e nefrologico; trova altresì impiego in situazioni di emergenza clinica come tecnica di detossificazione sia endogena che esogena, dove l'intervento di rimozione si rende necessario prima che si sia instaurato un danno d'organo irreversibile (14-18). La terapia aferetica trova elettiva indicazione nelle fasi di acuzie consentendo una più rapida ripresa organica e funzionale nel trattamento precoce, ma in rari casi può assumere il ruolo di una terapia cronica $(8,9,16)$. Secondo i criteri dell'ASFA, le patologie che possono essere classificate nelle seguenti quattro categorie sulla base della riconosciuta efficacia della terapia aferetica stessa:

categoria I: patologie in cui l'aferesi è riconosciuta come terapia di prima scelta sulla base di trial controllati;

categoria II: patologie in cui l'aferesi può essere utilizzata come terapia di supporto;

categoria III: patologie in cui non sono disponibili trial controllati per supportare la validità dell'aferesi;

categoria IV: patologie in cui l'aferesi non dà benefici. 
Nella Tabella I vengono riportate le dieci patologie più frequentemente trattate nel mondo con aferesi terapeutica $(8,9,11)$.

\section{Analisi dei costi}

Un'attenta disamina sull'analisi dei costi della terapia aferetica deve prendere in considerazione una serie di variabili che includono, oltre ai costi effettivi dei materiali utilizzati per la tecnica, anche la scelta della metodica più appropriata, l'efficacia della stessa e la possibilità che il tempestivo utilizzo di questa terapia possa prevenire l'instaurarsi di lesioni irreversibili e quindi portare al totale recupero sociale e lavorativo del paziente e a una riduzione dell'invalidità, delle giornate di ricovero e dei farmaci. Ciascuna delle metodiche aferetiche ha applicazioni, potenzialità e costi diversi. Le metodiche selettive di immunoadsorbimento hanno per esempio un costo maggiore, mentre la doppia filtrazione a cascata risulta probabilmente, alla pari del plasma-exchange classico, la più economica. Le metodiche utilizzate e i relativi costi variano altresì da Paese a Paese in rapporto, perlopiù, con la politica sanitaria della Nazione. In Italia, esistono differenze anche nell'ambito delle diverse Regioni. Nel "costo totale" di una determinata prestazione vanno sempre tenuti in considerazione, oltre ai costi quantificabili (costo "hardware", costo di gestione, ecc.), anche un costo "sociale", che risulta di più difficile quantificazione. Per il costo di gestione dovranno essere conteggiati il personale impiegato (qualifica, numero, tempo di lavoro), i materiali utilizzati (costo unitario e quantità) e le apparecchiature utilizzate (costi delle apparecchiature e della manutenzione delle stesse in rapporto con il numero di prestazioni effettuabili in un anno). A ciò bisogna aggiungere una quota che inglobi i costi generali della struttura erogatri$c e$, non legati ai singoli trattamenti, e quelli relativi al funzionamento generale della struttura di appartenenza dell'unità produttiva (Tab. II). Il costo sociale risulta influenzato dall'efficacia della terapia aferetica e non va inteso come aggravio

TABELLA I - PATOLOGIE TRATTATE CON AFERESI TERAPEUTICA DIVISE PER CONTINENTI

\begin{tabular}{|c|c|c|c|c|c|c|c|}
\hline Patologia & Tecnica Aferetica & Classe Asfa & Asia & Europa & Nord America & $\begin{array}{c}\text { Centro-Sud } \\
\text { America }\end{array}$ & $\begin{array}{c}\text { Pazienti Trattati } \\
\text { Per Patologia }\end{array}$ \\
\hline Miastenia Gravis & Plasma-exchange & I & 81 & 30 & 22 & 3 & 136 \\
\hline Epatopatie & Plasma-exchange & III & 40 & & & & 40 \\
\hline Artrite Reumatoide & Immunoadsorbimento & II & 38 & & & & 38 \\
\hline LES & Plasma-exchange & III & 33 & 6 & & & 39 \\
\hline Nefrite Lupica & Plasma-exchange & IV & 38 & & & & 38 \\
\hline S. Guillan-Barrè & Plasma-exchange & I & 26 & 27 & 15 & 4 & 72 \\
\hline Colite Ulcerosa & Adoptive cytapheresis & $\mathrm{P}$ & 20 & & & & 20 \\
\hline Pemfigo Volgare & $\begin{array}{l}\text { Plasma-exchange } \\
\text { Extracorporeal } \\
\text { photopheresis }\end{array}$ & $\begin{array}{l}\text { III } \\
\text { III }\end{array}$ & 17 & & & & 17 \\
\hline Patologie Infettive & Plasma-exchange & III & 16 & & & & 16 \\
\hline \multirow[t]{2}{*}{ Ipercolesterolemia } & Selective removal & II & & 40 & & & \\
\hline & Plasma-exchange & II & 16 & & & & 56 \\
\hline Sclerodermia & $\begin{array}{l}\text { Plasma-exchange } \\
\text { Extracorporeal } \\
\text { photopheresis }\end{array}$ & $\begin{array}{l}\text { III } \\
\text { IV }\end{array}$ & & 28 & & & 28 \\
\hline Altre Vasculopatie & Plasma-echange & III & & 21 & & 2 & 23 \\
\hline Glomerulonefriti & Plasma-exchange & III & & 17 & & & 17 \\
\hline Crioglobulinemia & Plasma-exchange & I & & 12 & 8 & & 20 \\
\hline M. Di Buerger & & & & 9 & & & 9 \\
\hline Polineuropatie Recidevanti & Plasma-exchange & I & & 6 & & & 6 \\
\hline Neoplasie & $\begin{array}{l}\text { Extracorporeal } \\
\text { photopheresis }\end{array}$ & III & & & 36 & & 36 \\
\hline Sclerosi Multipla & Plasma-exchange & III & & & 24 & & 24 \\
\hline Seu & Plasma-exchange & III & & & 7 & & 7 \\
\hline PTT & Plasma-exchange & I & & & & 3 & 3 \\
\hline Trombocitosi & Trombo-cytapheresis & II & & & 6 & 3 & 9 \\
\hline \multirow[t]{2}{*}{ Itp } & Immunoadsorbimento & II & & & & & \\
\hline & Plasma-exchange & IV & & & & 2 & 2 \\
\hline \multicolumn{3}{|c|}{ Totale pazienti trattati per continente } & 325 & 196 & 118 & 17 & 656 \\
\hline
\end{tabular}


di spesa bensì come potenziale risparmio legato al recupero sociale e lavorativo del soggetto. La tempistica dell'intervento terapeutico è fondamentale. In diversi Paesi la terapia aferetica viene infatti prescritta per determinate malattie solo in caso di mancata risposta alla terapia farmacologica e in condizioni cliniche estremamente gravi. La terapia aferetica può quindi risultare poco valida se attuata tardivamente, dopo che terapie farmacologiche, spinte all'estrema ratio, abbiano fallito, lasciando i danni derivanti dagli effetti collaterali delle stesse e senza possibilità di una "restitutio ad integrum". Potremmo auspicare l'individuazione di "fasce di prescrizione" $\mathrm{o}$, meglio, come avviene per la terapia dialitica, i cui costi incidono notevolmente sul SSN, di diversificare nell'utilizzo le varie tecniche. Alla luce di queste considerazioni, le metodiche aferetiche possono risultare nel loro insieme un presidio terapeutico non più oneroso di una terapia farmacologica a lungo termine $(20,21)$.

\section{Possibili sviluppi tecnologici}

La plasmaferesi terapeutica ha subito negli anni un cambiamento notevole, conseguente allo sviluppo tecnologico delle apparecchiature e a un'espansione delle indicazioni. Infatti, l'innovazione tecnologica ha introdotto metodiche che permettono un trattamento più tollerabile e meno invasivo. Recentemente sono stati introdotti in Europa dei sistemi che utilizzano dei sofi- sticati filtri in grado di ridurre i rischi legati alla procedura, ampliando, dove possibile, le indicazioni $(8,11)$.

Il futuro dell'aferesi terapeutica sembra rivolto verso la plasmaperfusione a cascata, l'immunoadsorbimento e l'ECP, oltre che verso la nanotecnologia. In questa prospettiva si inserisce la tecnologia Hemofenix. Al contrario dei sistemi tradizionali di plasmaferesi per centrifugazione, il dispositivo Hemofenix utilizza un sistema di nanofiltrazione. La separazione del sangue tramite plasmaferesi "Hemofenix", che utilizza la filtrazione mediante membrana, permette la separazione del plasma durante il passaggio del sangue attraverso un particolare tipo di filtro, una membrana nanotecnologica, porosa e piatta. Il plasma-filtro ROSA della Hemofenix è costituito dalla sovrapposizione di più membrane porose (membrane Track), ricoperte da nano-pori. Le membrane sono costituite da un film polimerico sottile con spessore di 10-15 $\mu$, costituito da polietilentereftalato o policarbonato, con un numero di pori per cm quadrato pari a $105-3 \times 109 \mathrm{~cm}^{2}$, ciascuno con un diametro che varia in un range di 0.05-5.0 $\mu \mathrm{m}$. La membrana Track è costituita da un elevato numero di strati, legati tra loro con una speciale tecnica e poi posizionati in condizioni sterili all'interno di un contenitore di plastica blu delle dimensioni di $10 \times 10 \mathrm{~cm}$. La realizzazione viene effettuata in Russia attraverso un ciclotrone in grado di produrre membrane delle dimensioni di 30-50 nanometri $(21,22)$.

Le principali proprietà delle membrane Track sono le seguenti:

TABELLA II - COSTO MEDIO DELLA PROCEDURA AFERETICA

\begin{tabular}{|c|c|c|c|c|c|c|}
\hline \multirow[b]{2}{*}{ Tipo Di Procedura } & \multicolumn{2}{|c|}{ Costo Hardware } & \multicolumn{2}{|c|}{ Costo Di Gestione } & \multirow[b]{2}{*}{ Costo Tot. } & \multirow[b]{2}{*}{$\begin{array}{l}\text { H impegno } \\
\text { medio }\end{array}$} \\
\hline & $\begin{array}{l}\text { Materiali } \\
\text { di consumo }\end{array}$ & $\begin{array}{l}\text { Liquidi } \\
\text { di sostituzione } \\
\text { e farmaci }\end{array}$ & Costi vari & $\begin{array}{c}\text { Costo } \\
\text { personale }\end{array}$ & & \\
\hline & $€$ & $€$ & $€$ & $€$ & $€$ & \\
\hline PE per centrifugazione & 186.58 & 356.00 & 50.00 & 200.00 & 792.58 & 5 \\
\hline Hemofenix Nanofiltrazione & 150.00 & 15.00 & 50.00 & 100.00 & 315.00 & 2 \\
\hline PE per filtrazione & 175.07 & 356.00 & 50.00 & 200.00 & 781.07 & 5 \\
\hline Plasmaperfusione su cascata & 404.89 & 85.00 & 50.00 & 200.00 & 739.89 & 5 \\
\hline $\begin{array}{l}\text { Adsorbimento con legame fisico- } \\
\text { chimico (IM-TR350, PH350, BR350) }\end{array}$ & 928.07 & 20.00 & 50.00 & 200.00 & 1198.07 & 5 \\
\hline $\begin{array}{l}\text { Adsorbimento con legame biologico } \\
\text { (prot. A stafilococcica) } \\
\text { (Immusorba) } \\
\text { (Prosorba) }\end{array}$ & $\begin{array}{c}(11.000 / 10 \\
\text { tratt. teorici) } \\
1275.07 \\
1826.00\end{array}$ & $\begin{array}{l}20.00 \\
20.00\end{array}$ & $\begin{array}{l}50.00 \\
50.00\end{array}$ & $\begin{array}{l}200.00 \\
200.00\end{array}$ & $\begin{array}{l}1545.07 \\
2096.00\end{array}$ & $\begin{array}{l}5 \\
5\end{array}$ \\
\hline $\begin{array}{l}\text { Adsorbimento con legame immuno- } \\
\text { logico (LDL-aferesi + IM-Ig sistema } \\
\text { Therasorb-Moltenyi) }\end{array}$ & $\begin{array}{l}(16.000 / 40 \\
\text { tratt. teorici) } \\
588.64\end{array}$ & 470.00 & 50.00 & 200.00 & 1308.64 & 5 \\
\hline Sistema HELP & 884.06 & 20.00 & 50.00 & 200.00 & 1154.06 & 5 \\
\hline Sistema Dali & 802.06 & 20.00 & 50.00 & 200.00 & 1072.06 & 5 \\
\hline Sistema Kaneka (Liposorb, Selesorb) & 1293.20 & 20.00 & 50.00 & 200.00 & 1563.20 & 5 \\
\hline Fotochemioterapia Ex. (ECP) & 878.75 & 60.00 & 50.00 & 200.00 & 1188.75 & 5 \\
\hline
\end{tabular}


- elevata selettività

- inerzia biologica

- superficie della pellicola liscia (ciò contribuisce a un migliore passaggio del sangue attraverso i canali del filtro con una minore pressione per filtrare il sangue)

- bordi dei pori lisci (il passaggio del sangue attraverso la membrana non provoca traumi agli elementi cellulari)

- elevata uniformità della superficie porosa

- elevata uniformità del diametro dei pori

- stabilità

Il sangue intero che arriva al filtro ROSA riempie la prima camera (camera sangue) dove si trovano le membrane filtranti e passa poi nella camera per il plasma dove viene separato dagli elementi corpuscolati. La bassa resistenza al flusso del sangue attraverso le camere è utile in quanto migliora la stabilità del flusso ematico; infatti tale tecnologia previene il traumatismo delle cellule ematiche.

Il sistema Hemofenix consente di effettuare il trattamento in monoago con un ridotto volume extracorporeo, di circa $70 \mathrm{~mL}$. Ciò limiterebbe i rischi per i pazienti instabili dal punto di vista cardiovascolare e ne permetterebbe l'utilizzo nei bambini, anche neonati, così come nei pazienti che presentano sintomi di debilitazione come gli anziani. I bambini possono presentare maggiori rischi e complicanze, pertanto devono essere attentamente valutati i rischi del posizionamento di un accesso vascolare e le corrette indicazioni per la tecnica di aferesi.

\section{TABELLA III - HEMOFENIX ROSA}

\begin{tabular}{|c|c|}
\hline $\begin{array}{l}\text { Vantaggi del filtro per il plasma } \\
\text { ROSA }\end{array}$ & $\begin{array}{l}\text { Caratteristiche principali del filtro } \\
\text { per il plasma ROSA }\end{array}$ \\
\hline $\begin{array}{l}\text { Processo di filtrazione } \\
\text { stabile grazie al rivestimento } \\
\text { protettivo rigido del filtro }\end{array}$ & $\begin{array}{l}\text { Capacità di separazione del plasma } \\
\text { durante il processo di filtrazione } \\
\text { superiore a } 15 \mathrm{~mL} / \mathrm{min}\end{array}$ \\
\hline $\begin{array}{l}\text { - Riduzione del traumatismo } \\
\text { degli eritrociti e di altri } \\
\text { elementi cellulari plasmatici }\end{array}$ & $\begin{array}{l}\text { Volume di riempimento infe- } \\
\text { riore a } 20 \mathrm{~mL} \\
\text { - Peso: } 100 \mathrm{mg}\end{array}$ \\
\hline $\begin{array}{l}\text { Rispetto dei requisiti della } \\
\text { Commissione della Farmaco- } \\
\text { pea Europea e del Comitato } \\
\text { per la Sanità Pubblica del } \\
\text { Consiglio Europeo }\end{array}$ & - Dimensioni: $85 \times 85 \times 35 \mathrm{~mm}$ \\
\hline
\end{tabular}

\section{TABELLA IV - PROCEDURA DELLA PLASMAFERESI ROSA SENZA DISPOSITIVO HEMOFENIX}

\begin{tabular}{ll}
\hline $\begin{array}{l}\text { Principali caratteristiche della } \\
\text { procedura della plasmaferesi ROSA } \\
\text { senza dispositivo Hemofenix }\end{array}$ & $\begin{array}{l}\text { Vantaggi della procedura della pla- } \\
\text { smaferesi ROSA senza dispositivo } \\
\text { Hemofenix }\end{array}$ \\
$\begin{array}{l}\text { Velocità di ricezione del plasma } \\
\text { fino a } 600 \mathrm{~mL} / \text { ora }\end{array}$ & $\begin{array}{l}\text { Autonomia e indipendenza da fonti } \\
\text { di energia elettrica }\end{array}$ \\
$\begin{array}{l}\text { Dispositivo sterile, ignifugo e non } \\
\text { tossico }\end{array}$ & $\begin{array}{l}\text { Requisiti logistici per il suo } \\
\text { trasporto minimi } \\
\end{array}$ \\
Rapidità di funzionamento
\end{tabular}

Tutte le metodiche di aferesi, come è noto, richiedono un accesso vascolare adeguato, utilizzando, talvolta, cateteri venosi centrali oppure fistole artero-venose già esistenti. Nel caso della tecnologia Hemofenix l'accesso vascolare per la circolazione extracorporea può avvenire tramite un'unica via d'accesso da una vena periferica, in genere la vena cubitale (ago $18 \mathrm{G})$, minimizzando il traumatismo stesso delle vene.

La plasmaferesi con plasma-filtro ROSA è eseguita utilizzando Hemofenix con un flusso discontinuo e con accesso a un singolo ago; in caso di contemporanea infusione di liquido di sostituzione si ricorre a un secondo accesso vascolare indipendente e manuale.

La somministrazione dell'anticoagulante avviene attraverso un dispositivo di regolazione manuale e non dipende dalle variazioni dei parametri di perfusione.

Lo strumento risulta facilmente trasportabile e versatile per le sue dimensioni e per il suo peso ridotti, pertanto potrebbe essere utilizzato a livello ambulatoriale oppure in reparti come la terapia intensiva.

Nella Tabella III sono mostrate le principali caratteristiche e i principali vantaggi del plasma-filtro ROSA.

Il filtro ROSA consente un utilizzo indipendente dal dispositivo Hemofenix e potrebbe pertanto essere utilizzato da medici e paramedici in occasione di disastri per consentire l'assistenza ai feriti in situazioni di emergenza esterna (crush syndrome) (Tab. IV).

Il filtro ROSA potrebbe essere utilizzato in procedure di plasmaferesi mediante membrana per il trattamento di disintossicazioni in malattie croniche e acute, dietro opportuna indicazione e per la raccolta di plasma nei centri trasfusionali. I vantaggi che questa metodica potrebbe offrire, sono: un priming ridotto, un ridotto quantitativo di plasma rimosso, con un minore rischio infettivo e allergenico, e una minore durata del trattamento $(16,23,24)$.

Le procedure di aferesi terapeutica sono piuttosto sicure, ma richiedono comunque la presenza di personale esperto, specializzato e adeguatamente formato. Attualmente la plasmaferesi viene utilizzata sia in regime di urgenza che per il trattamento programmato di pazienti con patologie croniche, dietro opportuna indicazione, argomento che rimane molto controverso e dibattuto anche per lo scarso numero di pazienti trattati nelle casistiche internazionali.

\section{Riassunto}

Nel 1914, Abel, insieme a Rowentree e Turner, ha introdotto il termine "plasmaferesi", il cui significato letterale è "sottrazione". La prima "plasmaferesi terapeutica" risale al 1952 in un paziente affetto da mieloma multiplo, ma, nel 1963, iniziarono le prime applicazioni cliniche per ridurre l'iperviscosità del sangue in pazienti affetti da paraproteinemia, ad opera di Salomon e Fahey. Nel tempo sono state introdotte tecniche sempre più specifiche e selettive, ampliando notevolmente le indicazioni cliniche (plasmaexchange, crioaferesi, leucoaferesi, trombocitoaferesi, linfocitoaferesi LDL aferesi). Le attuali indicazioni alla plasmaferesi vengono definite e periodicamente ristabilite 
da due associazioni scientifiche americane, l'American Association of Blood Banks (AABB) e l'American Society of Apheresis (ASFA), sulla base delle prove di efficacia del trattamento nelle malattie specifiche. Nel 1993 è stato costituito, nell'ambito della Società Italiana di Nefrologia, il gruppo di studio dell'aferesi terapeutica che ha il compito di sviluppare Linee Guida di riferimento per il trattamento con plasmaferesi. Il fine ultimo della terapia aferetica sarebbe quello di poter rimuovere dal circolo solo le sostanze patogene, ma l'utilizzo di tecniche di rimozione selettiva si accompagna in realtà non tanto a una maggiore capacità di estrazione della sostanza, bensì a una minore rimozione di componenti non patologiche, riducendo il rischio di infezioni, emorragie e reazioni allergiche. Tuttavia, la plasmaferesi potrebbe anche agire modulando il sistema immunitario oltre che rimuovendo le sostanze patogene. L'aferesi terapeutica è indicata in immunologia, dermatologia, ematologia, oncologia e nelle malattie dismetaboliche, neurologiche e renali ed è utilizzata anche nelle emergenze come tecnica di detossificazione sia endogena che esogena, in cui è necessaria la rimozione della sostanza patogena prima che si verifichi un danno d'organo irreversibile. La plasmaferesi terapeutica ha subito negli anni un cambiamento notevole conseguente allo sviluppo tecnologico delle apparecchiature e a un'espansione delle indicazioni. Infatti, l'innovazione tecnologica ha introdotto metodiche che permettono un trattamento più tollerabile e meno invasivo. Hemofenix utilizza la filtrazione mediante membrana attraverso un sistema di nanofiltrazione, il filtro ROSA. Hemofe- nix, permettendo di eseguire il trattamento con un singolo e piccolo ago e con un volume extracorporeo ridotto, circa 70 $\mathrm{mL}$, potrebbe ridurre i rischi per il paziente, anche pediatrico. Ulteriori vantaggi potrebbero essere rappresentati dalla breve durata del trattamento e dalla mancata necessità di utilizzare il plasma come fluido di sostituzione, riducendo il rischio di infezioni e reazioni allergiche. Sicuramente, oltre alla sicurezza, dovrà essere valutata la reale efficacia in trial clinici randomizzati, confrontando questa metodica con le terapie aferetiche classiche.

Parole chiave: Aferesi terapeutica, Nanofiltrazione, $\mathrm{He}$ mofenix, Filtro ROSA

Dichiarazione di conflitto di interessi: Gli Autori dichiarano di non avere conflitto di interessi.

Contributi economici agli Autori: Gli Autori dichiarano di non aver ricevuto sponsorizzazioni economiche per la preparazione dell'articolo.

Indirizzo degli Autori:

Prof. Gaspare Elios Russo

Via Buster Keaton, 12

00128 Roma

gasparelios.russo@uniroma1.it

\section{Bibliografia}

1. Kolins J, Jones JM Editors. Therapeutic Apheresis Technical Workshop. Arlington, Virginia, American Association of Blood Banks I, 3-13; II, 15-23; 1983.

2. Gurland HJ, Lysaght MJ, Samtleben W, Schmidt B. Comparative evaluation of filter used in membrane plasmapheresis. Nephron 1984; 36: 173-82.

3. Greinix HT, Volc-Platzer B, Kalhs P, et al. Extracorporeal photochemotherapy in the treatment of severe steroid-refractary acute graft versus host disease: a pilot study. Blood 2000; 96 (7): 2426-31.

4. Child FJ, Ratnavel R, Russel-Jones R, et al. Extracorporeal Photopheresis (ECP) in the treatment of chronic graft-versus-host disease (GVHD). Bone Marrow Transplant 2000; 25 (5): 575.

5. Russo GE, Tosco U, Torre MC, et al. La plasmaferesi (PF) nel LES: plasma-exchange filtrazione a cascata? Atti IV Congr. Naz. Soc. It. di Emaferesi. S. Margherita Ligure (GE), 8-1O Marzo 1989.

6. Ronco C, Brendolan A, Lonnemann G, et al. A pilot study of plasmafiltration with adsorption in septic shock. Crit Care Med 2002; 30: 1250-5.

7. Formica M, Olivieri C, Livigni S, et al. Hemodynamic response to couplet plasmafiltration adsorption in human septic shock. Intensive Care Med 2003; 29: 703-8.

8. Malchesky PS, Koo AP, Skibinski CI, Hadsell AT, Rybicki LA. Apheresis technologies and clinical applications: the 2007 International Apheresis Registry. Ther Apher Dial 2010; 14 (1): 52-73.

9. Szczepiórkowski ZM, Bandarenko N, Kim HC, et al. Guidelines on the use of therapeutic apheresis in clinical practice: evidence-based approach from the Apheresis Applications Committee of the American Society for Apheresis. J Clin Apher 2007; 22: 106-75.

10. Szczeklik W, Mitka I, Novak I, et al. Plasmapheresis in intesive care units. Anaesth Int Ther 2010; 42 (2): 90-5.

11. Schwartz J, Winters JL, Padmanabhan A, et al. Guidelines on the use of therapeutic apheresis in clinical practice-evidencebased approach from the Writing Committee of the American Society for Apheresis: the sixth special issue. J Clin Apher 2013; 28 (3): 145-284.

12. Ronco C. Endotoxin removal: history of a mission. Blood Purif 2014; 37 (Suppl. 1): 5-8.

13. Pusey CD, Levy JB. Plasmapheresis in immunologic renal disease. Blood Purif 2012; 33 (1-3): 190-8.

14. Ramírez-Bueno A, Salazar-Ramírez C, Cota-Delgado F, de la 
Torre-Prados MV, Valdivielso P. Plasmapheresis as treatment for hyperlipidemic pancreatitis. Eur J Intern Med 2013.

15. Samtleben W, Mistry-Burchardi N, Hartmann B, Lennertz A, Bosch T. Therapeutic plasma exchange in the intensive care setting. Ther Apher 2001; 5 (5): 351-7.

16. Nenov VD, Marinov P, Sabeva J, Nenov DS. Current applications of plasmapheresis in clinical toxicology. Nephrol Dial Transplant 2003; 18 (Suppl. 5): v56-8.

17. Shaz BH, Linenberger ML, Bandarenko N, et al. Category IV indications for therapeutic apheresis: ASFA fourth special issue. J Clin Apher 2007; 22 (3): 176-80.

18. Sarode R, Bandarenko N, Brecher ME, et al. Thrombotic thrombocytopenic purpura: 2012 American Society for Apheresis (ASFA) consensus conference on classification, diagnosis, management, and future research. J Clin Apher 2013.

19. Russo GE, Bauco B, De Paola A, Vitaliano E, Bonello M. Prin- cipali campi di applicazione dell'Aferesi Terapeutica. Atti V Corso di Aggiornamento in Emaferesi e Autotrasfusione. In: Progressi in Emaferesi. Edizioni S.I.d.E.S., Roma 2000, vol. V; 63-74.

20. Russo GE, et al. Therapeutical apheresis: cost analysis. The International Journal of Artificial Organs 1998; 21 (Suppl. 6): 72-7.

21. Voĭnov AV, Bedrov AIa, Voinov VA. Syndrome of "diabetic foot". Vestn Khir Im I I Grek 2012; 171 (3): 106-9.

22. Voinov VA. Strategy of efferent therapy in sepsis. Vestn Khir Im I I Grek 2013; 172 (2): 74-7.

23. Goldstein SL. Therapeutic apheresis in children: special considerations. Semin Dial 2012; 25 (2): 165-70.

24. Shemin D, Briggs D, Greenan M. Complications of therapeutic plasma exchange: a prospective study of 1,727 procedures. $\mathrm{J}$ Clin Apher 2007; 22 (5): 270-6. 